\title{
CDW and CDW-EIS Calculations for FDCSs in Highly Charged Ion Impact Ionization of Helium
}

\author{
M. Ciappina and W. R. Cravero \\ Av. Alem 1253, (8000) Bahía Blanca, Argentina
}

Received on 29 July, 2005

\begin{abstract}
In this work we present fully differential cross sections (FDCS) calculations using CDW and CDW-EIS theories for helium single ionization by $100 \mathrm{MeV} / \mathrm{amu}$ and $2 \mathrm{MeV} / \mathrm{amu} \mathrm{C}^{6+}$ and $3.6 \mathrm{MeV} / \mathrm{amu} \mathrm{Au}{ }^{24+}$ and $\mathrm{Au}^{53+}$ ions. We performed our calculations for different momentum transfers and ejected electron energies. We study the influence of the internuclear potential on the ejected electron spectra. We discuss different regimes where the internuclear interaction can or cannot be neglected. We compare our calculations with experimental data available. It is shown that for high impact energy and small momentum transfer, internuclear potential effects can be neglected in FDCSs.
\end{abstract}

Keywords: CDW; CDW-EIS; FDCS

\section{INTRODUCTION}

The study of electron emission spectra in ion-atom collisions has been a field of intense activity for years [1]. For intermediate to high energy single ionization there has been considerable theoretical efforts focused in the so-called two centre electron emission (TCEE) [2]. Improvement in the description of the ionized electron moving in the presence of both residual target and projectile fields after the collision (final state) has been a key aspect for the correct description of experimental data [3].

Within distorted wave approximations, it has been shown that, at least for high impact energy and multiply charged projectiles, the CDW theory of Belkić [4], used together with an appropiate description of the initial bound and final continuum electron states, yields best results for doubly differential cross sections (DDCSs) [5]. However, when the projectile impact velocity decreases, the CDW-EIS theory of Crothers and McCann [6] gives better results, its only difference being the choice of the initial state. Moreover the CDW-EIS is formally free of criticisms regarding the initial state proper normalization, and the transition amplitudes have not the divergent behavior that CDW exhibits (although it has been demonstrated that the CDW amplitudes are integrable and its DDCSs are well behaved [7]).

The field has now a renewed interest as a result of the development of the COLTRIMS (cold target recoil ion momentum spectroscopy) technique [8]. With COLTRIMS, the projectile tiny scattering angle can be obtained indirectly by measuring the ionized electron and recoil ion momenta [9]. Fully differential cross sections for ion impact ionization can now be measured and constitute a challenging ground for existing theories [10].

Fischer et al. [11] have reported absolute experimental measurements for $2 \mathrm{MeV} / \mathrm{amu} \mathrm{C}^{6+}$ single ionization of helium in the scattering plane, i.e., that defined by the initial and final projectile momenta, for various momentum transfers and ejected-electron energies. Theoretical results reported by these authors using a CDW-EIS model exhibited differences between experiment and theory on an absolute scale for emis- sion in the scattering plane [11]. Their calculations were made using the active electron approximation and hydrogenic wavefunctions for the initial and final states of the active electron [11]. Indeed, the simplest description for the He bound initial state is to assume it has one 'active' and one 'passive' electron and that the 'active' electron can be described as moving in the effective Coulomb field of the atomic core with an effective charge chosen (a) to reproduce the ionization energy or (b) so that the continuum wave is orthogonal to the initial state. A more sophisticated way is to apply a Hartree-Fock description for the initial state or to both initial and final states of the active electron [12]. However, Hartree-Fock wave functions do not include proper angular correlation between the two electrons in the helium target. Furthermore, for large perturbations, the incoming projectile may interact with more than one electron in a single collision event. An explicit two-electron description, i.e., a four-body theory might be necessary in that case. We have shown that by using the prior version of the usual CDW-EIS approximation together with an appropiate Roothan-Hartree-Fock description of the initial state and an effective charge coulomb wave function for the target electron continuum, we can get similar results to those obtained by using numerical Hartree-Fock wave functions in both channels [13], at least for DDCSs.

The aim of this paper is to present prior CDW and CDWEIS calculations with and without internuclear interaction taken into account for ion helium single ionization FDCSs at different perturbation regimes. Atomic units are used throughout unless otherwise stated.

\section{THEORIES}

We regard He single ionization as a single electron process and assume that in the final state the 'active' target electron moves in the combined Coulomb field of the target core with an effective charge $Z_{e f f}=1.345$ and the projectile field as considered within the CDW-EIS approach. For the initial bound state a Roothan-Hartree-Fock description is used. N-N interaction is treated as a pure Coulomb interaction between 




FIG. 1: FDCS for $100 \mathrm{MeV} / \mathrm{amu} \mathrm{C}^{6+}$ on He. Calculations in CDW and prior CDW-EIS,for different momentum transfer. Experimental data is from Ref. [9].

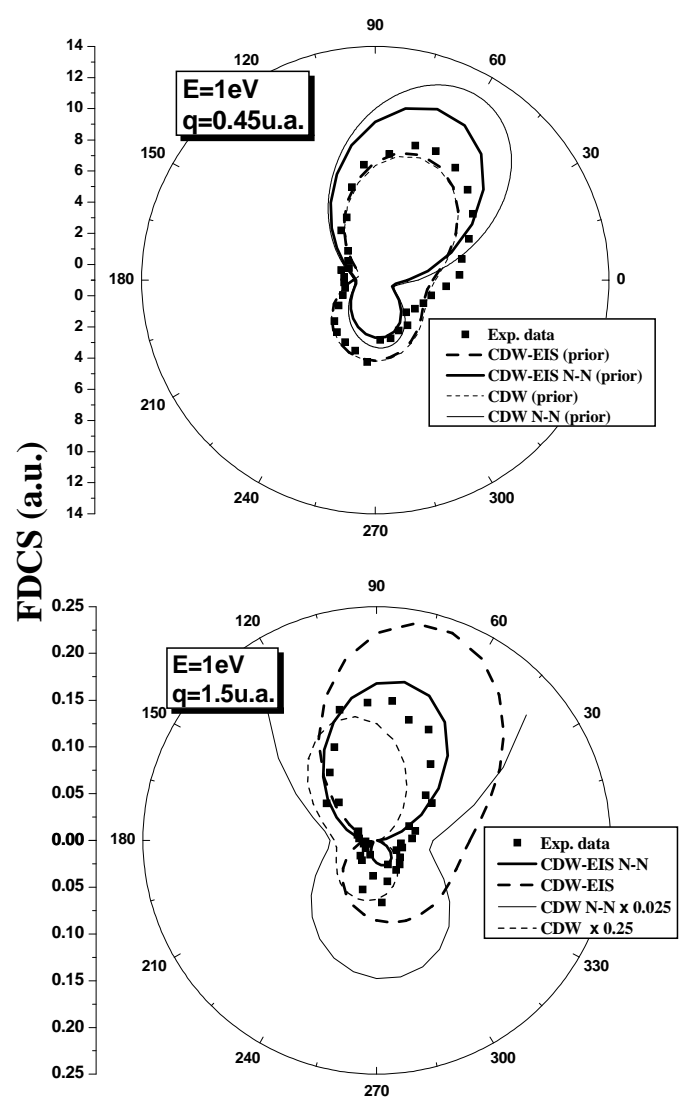

FIG. 2: FDCS for $2 \mathrm{MeV} / \mathrm{amu} \mathrm{C}^{6+}$ on He. Prior CDW and CDWEIS calculations with and without N-N interaction taken into account and for electron emission energy $1 \mathrm{eV}$. Experimental data is from Ref. [11].


FIG. 3: The same as in Figure 2 for electron emission energy $4 \mathrm{eV}$.

the projectile with a charge $Z p$ and the true target core charge, $Z_{T}=1$.

$\mathrm{N}-\mathrm{N}$ interaction can be taken into account in the transition amplitude $a_{i f}(\rho)$, in an eikonal approximation, through its multiplication by the corresponding phase factor [14], which for pure coulomb internuclear interaction results in [6]

$$
a_{i f}(\rho)=i(\rho v)^{2 i v^{\prime}} a_{i f}^{\prime}(\rho),
$$

where $v^{\prime}=\frac{Z_{P} Z_{T}}{v}, a_{i f}(\rho)\left(a_{i f}^{\prime}(\rho)\right)$ is the transition amplitude with (without) internuclear interaction. Using twodimensional Fourier transforms we have for the transition amplitude elements, as a function of the momentum transfer

$$
T_{i f}(\eta)=\frac{i v^{2 i v^{\prime}}}{(2 \pi)^{2}} \int d \eta^{\prime} T_{i f}^{\prime}\left(\eta^{\prime}\right) \int d \rho \rho^{2 \mathbf{i} v^{\prime}} e^{i\left(\eta-\eta^{\prime}\right) \cdot \rho} a_{i f}^{\prime}(\rho) .
$$

We solve the integral over the impact parameter analytically to obtain

$$
T_{i f}(\eta)=v^{\prime} \frac{i v^{2 i v^{\prime}}(2 \pi)^{-i v^{\prime}}}{2^{4} \pi^{3}} \int d \eta^{\prime} T_{i f}^{\prime}\left(\eta^{\prime}\right)\left|\eta-\eta^{\prime}\right|^{-2\left(1+i v^{\prime}\right)} .
$$


The remaining integral in Eq.(3) is evaluated numerically with an adaptive integration routine [15]. This approximation is valid as long as (i) the projectile suffers very small deflections in the collision and (ii) the velocity of the recoil ion remains negligible compared to that of the emitted electron.

The FDCS in energy and ejection angle of the electron and direction of the outgoing projectile is given by

$$
\frac{d \sigma}{d E_{k} d \Omega_{k} d \Omega_{K}}=(2 \pi)^{4} \mu^{2} k \frac{K_{f}}{K_{i}}\left|T_{i f}\right|^{2} \delta\left(E_{f}-E_{i}\right),
$$

where $\mu$ is the reduced mass of the proton-atom system, $K_{i}\left(K_{f}\right)$ is the magnitude of the incident particle initial (final) momentum and $T_{i f}$ is the corresponding transition amplitude.

The CDW approximation, was originally developed by Cheshire [16] and first applied by Belkić [4] for ion-atom ionization. Within this theory, the initial state distortion $\mathcal{L}_{i}^{+C D W}$ reads

$$
\mathcal{L}_{i}^{+C D W}\left(\mathbf{r}_{P}\right)=N\left(\alpha_{i}\right)_{1} F_{1}\left(i \alpha_{i} ; 1 ; i v_{P} r_{P}+i \mathbf{v}_{P} \cdot \mathbf{r}_{P}\right)
$$

with

$$
\alpha_{i}=\frac{Z_{P}}{v_{P}}
$$

and the corresponding short-range perturbation potential $W_{i}^{C D W} \chi_{i}^{+C D W}$ reads

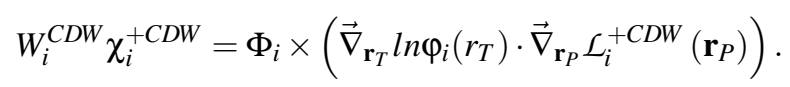

For the final state, we have

$$
\chi_{f}^{-C D W}(\mathbf{r}, t)=\Phi_{f}(\mathbf{r}, t) \times \mathcal{L}_{f}^{-C D W}\left(\mathbf{r}_{P}\right),
$$

where $\Phi_{i,(f)}$ is the unperturbed initial (final) state and the distortion $\mathcal{L}_{f}^{-C D W}$ reads

$$
\mathcal{L}_{f}^{-C D W}\left(\mathbf{r}_{P}\right)=N\left(\alpha_{P}\right)_{1} F_{1}\left(-i \alpha_{P} ; 1 ;-i k_{P} r_{P}-i \mathbf{k}_{P} \cdot \mathbf{r}_{P}\right),
$$

with $N\left(\alpha_{P}\right)$ being the usual Coulomb factor and

$$
\alpha_{P}=\frac{Z_{P}}{k_{P}}
$$

With this choice for the final wave function the Redmond asymptotic conditions for the three body Coulomb problem are satisfied [17]. In 1983, Crothers and McCann introduced the continuum distorted wave - eikonal initial state (CDW-EIS) for ion-atom ionization, which solved the problem of initial state normalization posed by CDW theory [6].

Within CDW-EIS initial state electron projectile distortion reads

$$
\mathcal{L}_{i}^{+E I S}\left(\mathbf{r}_{P}\right)=\exp \left(i v \ln \left(i v_{P} r_{P}+i \mathbf{v}_{P} \cdot \mathbf{r}_{P}\right)\right)
$$

while the perturbation potential is defined by
$W_{i}^{E I S} \chi_{i}^{+E I S}=\Phi_{i} \times\left(\frac{1}{2} \nabla_{r_{P}}^{2} \mathcal{L}_{i}^{+E I S}+\vec{\nabla}_{\mathbf{r}_{T}} \ln \varphi_{i}\left(r_{T}\right) \cdot \vec{\nabla}_{\mathbf{r}_{P}} \mathcal{L}_{i}^{+C D W}\right)$

In this paper we will use the prior version of CDW and CDW-EIS approximations which can be written as

$$
T_{i f}^{-C D W}=\left\langle\chi_{f}^{-C D W}\left|W_{i}\right| \chi_{i}^{+E I S}\right\rangle
$$

and

$$
T_{i f}^{-C D W-E I S}=\left\langle\chi_{f}^{-C D W}\left|W_{i}\right| \chi_{i}^{+E I S}\right\rangle
$$

respectively.

\section{RESULTS}

Using the theories outlined in the preceding section, we have performed calculations for different projectiles, spanning a large range of perturbation strengths as measured by the Bohr parameter $\kappa=2 Z_{P} / V_{P}$. In Fig. 1 we present results for $100 \mathrm{Mev} / \mathrm{amu} \mathrm{C}^{6+}$ single ionization of Helium calculated in prior CDW and prior CDW-EIS. Calculations with and without internuclear potential are practically identical in both cases. CDW-EIS calculations are in very good agreement with experiments, while CDW theory understimates data for low emision energy.

Figures 2 to 3 show results for $2 \mathrm{MeV} / \mathrm{amu} \mathrm{C}^{6+}$ single ionization at different momentum transfers. Here, while CDWEIS agrees reasonably well with experimental data, CDW fails to give the correct order of magnitude, and the angular behavior is less well reproduced. We observe that CDW-EIS calculation without N-N interaction gives better results for those momentum transfer that correspond, or are close to, "binary encounter" conditions, i.e., where the electron is ejected with a momentum close to that transferred by the projectile. We note, however, that $\mathrm{N}-\mathrm{N}$ interaction is not necessary to have a true three-body interaction during the collision process. The interaction of the outgoing electron with the residual target is taken into account in standard CDW or CDW-EIS calculations (even in FBA calculations, as long as coulomb wave functions are used for the ejected electron). Two-step scattering processes are accounted for, where the electron is first scattered by the incoming projectile and then bounce off the $\mathrm{He}^{+}$residual target ion. Internuclear potential opens a second "two-step" process in which part of the momentum is transferred to the active electron and then (or before) the projectile is scattered off the target nucleus. We realize that this semiclassical picture is more suitable for higher electron emission energy, and of course other purely quantum mechanisms do play an important role here. As projectile velocities are high and ejected electron velocity is small electrons may be screening N-N interaction so its inclusion should be more important for close collisions, or large momentum transfers, as it seems to be the case. Preliminary calculations show, however, that 
for emission energy above $10 \mathrm{eV}$ and small enough momentum transfers, internuclear potential plays again an important role and cannot be neglected. This would be in line with reports for DDCSs [18].

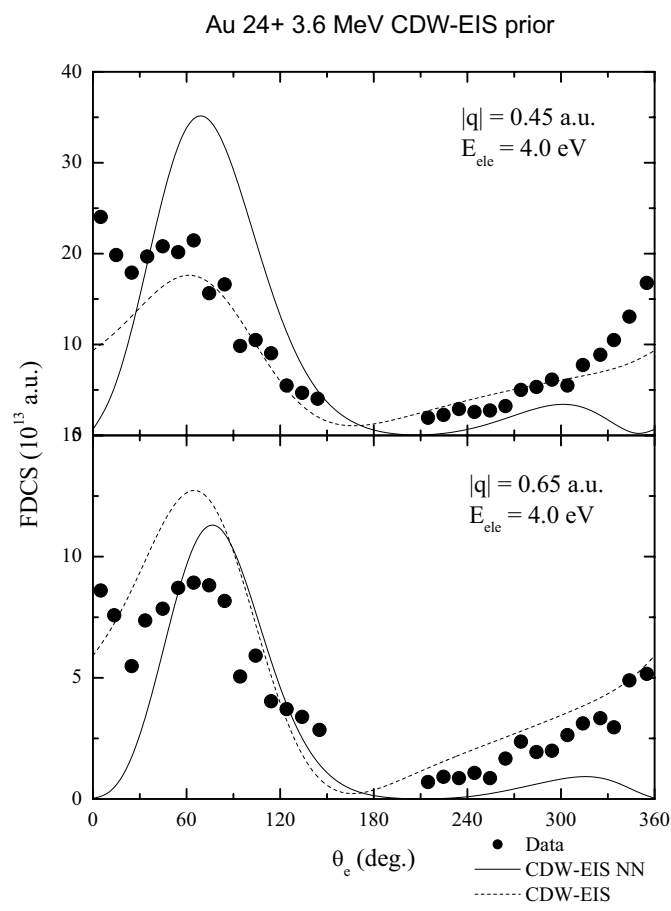

FIG. 4: $3.6 \mathrm{Mev} / \mathrm{amu} \mathrm{Au}{ }^{24+}$ on He. Prior CDW-EIS calculations with and without $\mathrm{N}-\mathrm{N}$ interaction taken into account for different momentum transfer and for electron emission energy $1 \mathrm{eV}$. Experimental data is from Ref. [11].

We find similar results for $3.6 \mathrm{MeV} / \mathrm{amu} \mathrm{Au}^{24+}$ and $\mathrm{Au}^{53+}$ impact ionization of He. In Figs. 4 to 7 we show CDW-EIS calculations for those systems with and without N-N interaction taken into account. Even when we are extending the validity range of the perturbative treatment $(\kappa \approx 4,8.8$ respectively), angular distribution is reasonably good. Yet, calculations with $\mathrm{N}-\mathrm{N}$ fail to yield the correct order of magnitude. We see again that for larger momentum transfer, internuclear interaction should be taken into account, while for smaller momentum transfer, calculations with and without N-N display similar angular distributions.

\section{CONCLUSIONS}

We have performed FDCSs calculations for highly charged ion impact ionization of Helium. We employed CDW and CDW-EIS theories taking into account N-N interaction but otherwise using as simple an approach as possible. The use of prior version helps us to avoid the need of more precise wave functions for the initial or final electronic states. We found reasonably good agreement with experimental data, even for Bohr parameters that put the system well outside the range of validity of a perturbative theory.

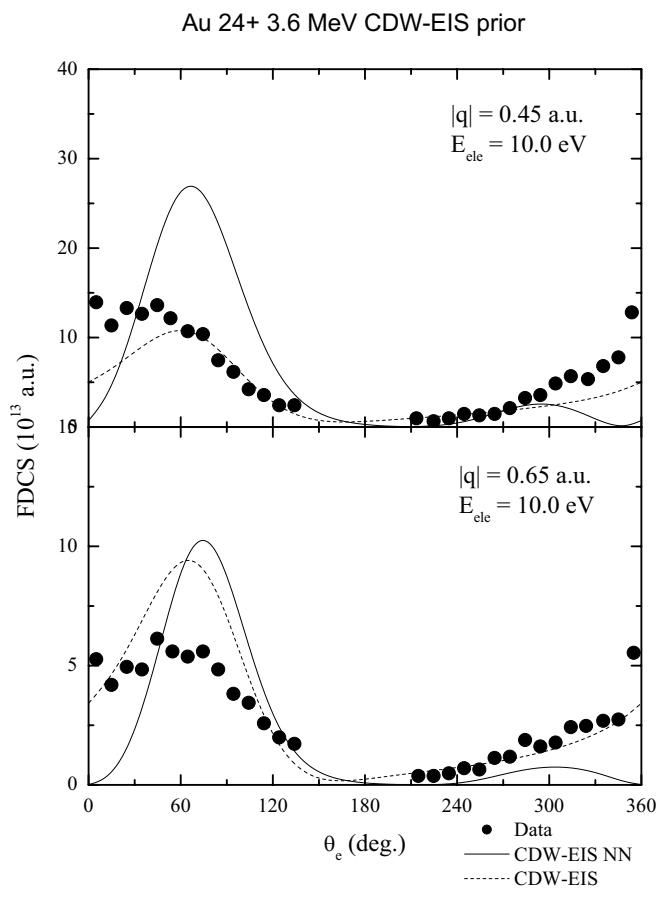

FIG. 5: The same as in Figure 4 for for electron emission energy 4 $\mathrm{eV}$.

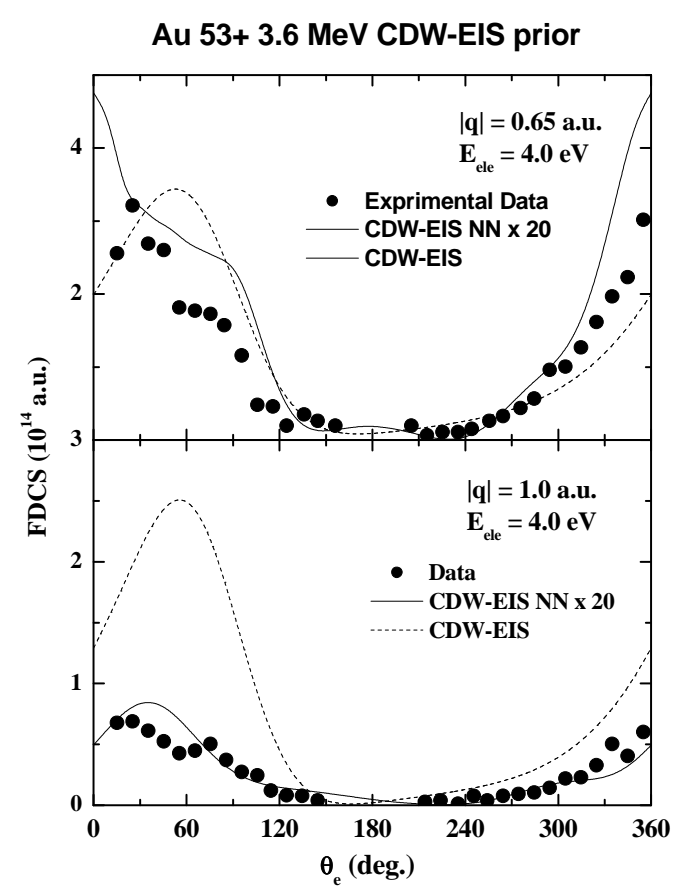

FIG. 6: $3.6 \mathrm{Mev} / \mathrm{amu} \mathrm{Au}^{53+}$ on He. Prior CDW-EIS calculations with and without $\mathrm{N}-\mathrm{N}$ interaction taken into account for different momentum transfer and for electron emission energy $4 \mathrm{eV}$. Experimental data is from Ref. [11]. 




FIG. 7: The same as in Figure 6 for electron emission energy $10 \mathrm{eV}$.
We see that, for small momentum transfers and ionized electron energies, the experimental data are well described by the CDW and CDW-EIS approximations without N-N interaction. However, for large momentum transfers, i.e., small impact parameters, other emission mechanisms involving projectile multiple scattering are enabled and the $\mathrm{N}-\mathrm{N}$ interaction plays an increasingly important role. Calculations also seem to show that $\mathrm{N}-\mathrm{N}$ interaction is important for larger electron energies and small momentum transfers. CDW calculations with $\mathrm{N}-\mathrm{N}$ interactions give poor results and generally do not yield the correct order of magnitude for the FDCSs.

Our calculations suggest that three-body dynamics as contained in standard prior CDW-EIS calculations seems to be enough in order to explain most of the structures observed for emission in the scattering plane. However, we note that for $\mathrm{Au}^{24+}$ and $\mathrm{Au}^{53+}$ projectiles the larger emission in the forward direction is not well reproduced by the theories.

\section{ACKNOWLEDGMENTS}

This work has been partially supported by Consejo Nacional de Investigaciones Científicas y Técnicas, Argentina, ANPCYT, PICT and Universidad Nacional del Sur under PGI 24/F027.
[1] N. Stolterfoht, R. D. DuBois, and R. D. Rivarola, Electron Emission in Heavy Ion-Atom Collisions (Springer, Berlin, 1997).

[2] J. O. Pedersen, P. Hvelplund, A. G. Petersen, and P. D. Fainstein, J.Phys. B: At. Mol. Phys. 23, L597 (1990).

[3] P. D. Fainstein, V. H. Ponce, and R. D. Rivarola, J. Phys. B: At. Mol. Opt. Phys. 24, 3091 (1991).

[4] Dz. Belkić, J. Phys. B: At. Mol. Phys. 11, 3529 (1978).

[5] L. Gulyás and P. D. Fainstein, J.Phys. B: At. Mol. Opt. Phys. 31, 3297 (1998).

[6] D. S. F. Crothers and J. F. McCann, J. Phys. B: At. Mol. Phys. 16, 3229 (1983).

[7] D. P. Dewangan and B. H. Bransden, J. Phys. B: At. Mol. Phys. 15, 4561 (1982).

[8] R. Moshammer, J. Ullrich, M. Unverzagt, W. Schmitt, P. Jardin, R. E. Olson, R. Mann, R. Dörner, V. Mergel, U. Buck, and H. Schmidt-Böcking, Phys. Rev. Lett. 73, 3371 (1994).

[9] M. Schulz, R. Moshammer, D. H. Madison, R. E. Olson, P. Marchalant, C. T. Welan, H. R. J. Walters, S. Jones, M. Foster, H. Kollmus, A. Cassimi, and J. Ullrich, J. Phys. B: At. Mol. Opt. Phys. 34, L305 (2001).
[10] M. Foster, D. H. Madison, J. L. Peacher, M. Schulz, S. Jones, D. Fischer, R. Moshammer, and J. Ullrich, J. Phys. B: At. Mol. Opt. Phys. 37, 1565 (2004).

[11] D. Fischer, R. Moshammer, M. Schulz, A. Voitkiv, and J. Ullrich, J. Phys. B: At. Mol. Opt. Phys. 36, 3555 (2003).

[12] L. Gulyás, P. D. Fainstein, and A. Salin, J. Phys. B: At. Mol. Opt. Phys. 28, 245 (1995).

[13] M. F. Ciappina, W. R. Cravero, and C. R. Garibotti, J.Phys. B: At. Mol. Opt. Phys. 36, 3775 (2003).

[14] R. McCarroll and A. Salin, J. Phys. B: At. Mol. Phys. 11, L693 (1978).

[15] H. Press, S. Teukolsky, W. Vetterling, and B. Flannery, Numerical Recipes in FORTRAN (Cambridge University Press, Cambridge, 1992).

[16] M. Cheshire, Proc. Roy. Soc. 84, 89 (1964).

[17] P. J. Redmond (unpublished), discussed in L. Rosenberg, Phys. Rev. D 8, 1833 (1973).

[18] P. D. Fainstein and L. Gulyás, J. Phys. B: At. Mol. Opt. Phys. 38, 317 (2005). 